\title{
DESARROLLO EMOCIONAL Y TRASTORNOS DEL ESPECTRO AUTISTA
}

\author{
Emotional development and autism spectrum disorders \\ CECILIA REYNA ${ }^{1}$ \\ Facultad de Psicología, Universidad Nacional de Córdoba, Argentina \\ (Recibido el 28/02/2011 - ACEPtAdo el 15/06/2011)
}

\begin{abstract}
RESUMEN
En este trabajo se revisan conceptos básicos del proceso emocional y su desarrollo durante la niñez, focalizándose en las particularidades que se manifiestan en los niños con trastorno del espectro autista. Se destaca la interrelación de la teoría de la intersubjetividad y la teoría de la mente, lo cual permite obtener una imagen más precisa de esta alteración.
\end{abstract}

Palabras clave: Emociones, autismo, desarrollo emocional.

\begin{abstract}
This paper reviews basic concepts of emotional process and its development during childhood, focusing on the particularities that occur in children with autism spectrum disorders. The interrelation between theory of intersubjectivity and theory of mind is emphasized; it allows obtaining a more accurate picture of this disorder.
\end{abstract}

Keywords: Emotions, autism, emotional development.

El término emoción comprende un proceso multidimensional que implica la interpretación subjetiva de situaciones significativas y la expresión o comunicación de emociones. El proceso emocional implica diversos componentes: a) experiencial, sentimientos que experimenta el sujeto, como alegría, tristeza e ira; b) observacional-motor, como expresiones faciales de sorpresa o miedo; y c) fisiológico, por ejemplo cambios en el ritmo cardíaco y aumento de sudoración (Cano, 1997; Fernández-Abascal, 2003).

En términos generales, el estudio de las emociones puede seguir dos direcciones: dimensional, debido a que existen diferencias individuales en las emociones que desarrollan las personas; y discrecional o específico, ya que hay características únicas y distintivas para cada categoría emocional. Algunas características de las emociones

1 Docente de la Facultad de Psicología, Universidad Nacional de Córdoba, Ciudad Universitaria, Argentina. E-mail: ceciliareyna@gmail.com 
discretas son la correspondencia entre el tipo de afrontamiento y la forma emocional, que tienen un procesamiento cognitivo propio y diferente a las otras emociones, y a su vez poseen una forma de expresión facial concreta y unívoca, de carácter universal (Fernández-Abascal, 2003).

Por otro lado, las emociones se pueden clasificar como primarias o secundarias. Las emociones primarias estarían ligadas a la maduración de los mecanismos y estructuras neurales que son las bases del proceso emocional y que, a lo largo del desarrollo facilitan la formación de patrones de evaluación y de respuesta a las emociones. Ejemplo de ellas son la alegría, la tristeza y la sorpresa. Por otra parte, las emociones secundarias son producto de la socialización y del desarrollo de capacidades cognitivas, como por ejemplo la culpa, la vergüenza, el orgullo y los celos. En este tipo de emociones influye la cultura en la que se desarrolla la persona y su propia historia personal (FernándezAbascal, 2003).

Desde distintas tendencias teóricas se intenta dar cuenta de la relación entre la aparición de las emociones en los niños y el desarrollo general. Algunas de las teorías propuestas son:

Teoría de las emociones (Izard, 1972). Desde el inicio de la vida existen algunas emociones básicas de carácter innato, que cuando el niño crece se van modificando y diferenciando, de tal manera que entre los 2 y 3 años se manifiestas emociones culturalmente reconocidas. Se considera que el desarrollo socio-emocional y cognitivo se influencian mutuamente, aunque, debido a que el sistema emocional es anterior, puede estimular el avance socio-cognitivo, por ejemplo, promoviendo las interacciones sociales, permitiendo reevaluar comportamientos o expectativas, o por medio de las representaciones sociales y el conocimiento. Pero a su vez, los avances que se producen a nivel socio-cognitivo estimulan el desarrollo emocional.

Teoría de los sistemas dinámicos (Fogel et al., 1992). Las emociones son procesos dinámicos que surgen debido a que el sujeto actúa en un contexto, los componentes innatos se ponen en juego a partir de la interacción con otras personas.

Análisis funcional de las emociones (Saarni, Mumme, \& Campor, 1998; Keltner \& Gross, 1999). Las emociones son producto de la adaptación a problemas del ambiente, permiten, por ejemplo, la formación del vínculo de apego, la evitación de daños físicos y la supervivencia, entre otros.

Abe e Izard (1999). Señalan hitos evolutivos en el desarrollo emocional y cognitivo. Inician el análisis en el nacimiento y finalizan en la adolescencia. A continuación se mencionarán los hitos principales que señalan estos autores entre los 0 y 12 años.

- $\quad$ De 0 a 2 años

- Aparición de las emociones primarias básicas: interés, placer, disgusto, tristeza, ira, sorpresa y miedo.

- Reconocimiento temprano (10 semanas) de expresiones faciales en los demás.

- Temperamento en un comienzo formado por el estilo de respuesta del bebé ante los estímulos del ambiente. 
A nivel evolutivo general aparecen las interacciones diádicas sincronizadas y la intersubjetividad primaria y secundaria. También surgen las conductas de referencia social (origen de la comprensión de estados mentales).

- $\quad$ De 2 a 5 años

- Consolidación de emociones primarias. Entra en juego la consideración de los estados mentales de los demás para entender la emoción.

- Miedos aprendidos, por ejemplo, miedo a la oscuridad o a los monstruos. La mayoría tiene su origen en la socialización de las emociones que realizan los padres.

- Emociones aprendidas como las rabietas. Muestran la necesidad de autonomía y autoafirmación.

- Aparición de emociones secundarias o sociales.

En este período surge la auto-conciencia, que se manifiesta en la expresión de emociones negativas y en conductas desafiantes. Hay una creciente sensibilidad hacia las normas sociales. Emergen de forma rudimentaria las emociones auto-evaluativas como culpa y la vergüenza. Un aspecto a destacar en este período es el incremento en la habilidad para comprender a los demás. Se observan conductas de empatía, conductas que manifiestan la comprensión de los estados mentales de los demás (como las creencias, deseos y emociones), es decir, se desarrolla la teoría de la mente (este punto se retomará luego).

- $\quad$ De 6 a 12 años

- Consolidación de las emociones auto-evaluativas debido a la internalización de las normas sociales y morales.

- Emociones contradictorias. El reconocimiento de la ambivalencia en un principio resulta difícil debido a que existe cierta tendencia a dicotomizar.

- Consolidación de la regulación emocional. Se aprende a regular sentimientos, cubrir emociones según la situación social. Esto evidencia no sólo la internalización de las reglas de expresión emocional en una sociedad, sino la capacidad para leer la mente de los demás.

En este período surge la habilidad para realizar comparaciones sociales debido a la mayor importancia del grupo de pares. Aparece el auto-concepto basado en rasgos estables de carácter. Se incrementa la habilidad para tomar una perspectiva social y para comprender los sentimientos y creencias de los demás.

Se ha reseñado brevemente lo que sucede en el desarrollo emocional "normal" durante la niñez. Pero, ¿qué sucede cuando el desarrollo está alterado? Uno de los casos prototípicos es el trastorno autista.

Desde la definición del autismo por Leo Kanner (1943), han proliferado las investigaciones sobre el autismo desde la neurobiología, la genética, la psicología y la psiquiatría, como no ha sucedido con ningún otro trastorno, o al menos en tal magnitud. Como señala Rivière 
(2004), la fascinación por el autismo se debe a que supone un desafío para algunas de las motivaciones más fundamentales como seres humanos. “... hay algo en la conducta autista que parece ir contra las leyes de gravedad entre las mentes...” (p. 330). Y poniéndose en el lugar de la persona con autismo la define como “... aquella persona a la cual las otras personas resultan opacas e impredictibles, aquella persona que vive como ausentes -mentalmente ausentes- a las personas presentes, y que por todo ello se siente incompetente para regular y controlar su conducta por medio de la comunicación...” (p. 329).

Esa dificultad para compartir el mundo (Rivière, 2001) se ha explicado desde dos teorías: la teoría de la intersubjetividad y la teoría de la mente. Desde la teoría de la intersubjetividad se considera que el autismo se debe a la carencia de habilidades afectivo-perceptuales básicas que son necesarias para relacionarse con otras personas. Se considera que ello explica su fallo en la comprensión de la mente de los otros, y a través de ello, la alteración de las capacidades para desarrollar un juego simbólico creativo y formas de lenguaje y pensamiento contextualmente apropiadas (Hobson, 1993). Según Trevarthen (1982), el acceso a la mente de los otros sería innata y el autismo sería un problema de la regulación por parte de las emociones del desarrollo cognitivo. Hobson (1993) también considera una base innata para relacionarse con las personas, siendo las emociones y los afectos los primeros caminos para acceder a la intersubjetividad, la cual permite captar la naturaleza mental de las personas.

Por otra parte, Baron-Cohen, et al. (1985) consideran al autismo como un trastorno cognitivo, que implica una alteración de la capacidad para atribuir mente e inferir estados mentales. Premack y Woodruff (1978) acuñaron el término "teoría de la mente" para dar cuenta de la habilidad de predecir, explicar e interpretar la conducta de los agentes en términos de estados mentales. La teoría de la mente no sólo es la capacidad para tener creencias como tales, sino para tener creencias sobre estados mentales, incluyendo la habilidad para tener creencias sobre creencias (Leslie, 2001). La teoría de la mente permite utilizar estrategias sociales, ya que posibilita, como señalan Rivière y Núñez (2001), "ponerse en la piel" del otro, "calzarse sus zapatos", dando lugar a la interpretación de las interacciones humanas no en términos netamente conductuales, sino en base a estados mentales. Es esa "mirada mental" (Rivière y Núñez, 2001) la que permite encontrarnos con la mente de los otros, interpretar su conducta y realizar predicciones, comprender que tienen deseos, creencias e intenciones, un mundo de emociones y experiencias diversas (Valdez, 2001).

Sin embargo, esa división entre las teorías no es tal como aparenta. El origen de la teoría de la mente implica un componente intersubjetivo de relaciones afectivas, las que no se desarrollan en un vacío, sino que requieren del desarrollo cognitivo de capacidades de inferencia y representación (Rivière, 2001).

Como se mencionó antes, Baron-Cohen et al. (1985) consideran que las personas con autismo tienen una alteración en la habilidad para comprender las representaciones mentales y su función en la acción del comportamiento de las personas, con dificultades para comprender que el comportamiento de la gente se da en base a creencias y deseos, y que la gente puede tener una base errada del mundo y actuar basados en esa mala representación; problemas en juegos de simulación e imaginación; y dificultades en los comportamientos que 
requieren atención conjunta como elaborar comportamientos comunicativos que requieren alguna comprensión de que uno puede hacer que una persona se de cuenta de los deseos de uno o perciba el interés de uno en aspectos específicos del mundo.

En 1983, Wimmer y Perner desarrollaron una tarea para evaluar el desarrollo de la teoría de la mente, la cual consistía en predecir la conducta del personaje de una historia atribuyéndoles una falsa creencia. Dicha tarea fue simplificada por el equipo de BaronCohen (1985). La historia comprende un personaje, Sally, que tiene una pelotita. La ubica en una canasta y la cubre, luego se retira. Mientras no está, entra Ann, encuentra la pelotita en la canasta y la mueve a una caja cercana. Luego vuelve a entrar Sally. El niño es interrogado directamente sobre la creencia de Sally: "¿Dónde cree Sally que está su pelotita?", para lo cual debe atribuir a Sally una creencia que es diferente a su propia creencia, y que el niño considera que es falsa. Finalmente es interrogado con dos preguntas control para evaluar la comprensión básica: "¿Dónde está ahora la pelotita?" y "¿Dónde estaba la pelotita el comienzo?". Ellos encontraron que el $80 \%$ de la muestra de niños con autismo contestaba erróneamente que Sally creía que la pelotita estaba en la caja. La mayor parte de los niños de cuatro años normales, como el $86 \%$ de los niños con síndrome de Down dijeron que Sally creía que estaba en la canasta. Esto dio lugar a considerar que las personas con autismo tenían un déficit específico para mentalizar.

Sotillo (2001) destaca el trabajo de investigadores que intentan dar cuenta de lo que ocurre antes de la solución de la tarea de la falsa creencia, como lo es el hecho de tratar de comprender lo que sucede a nivel de comprensión emocional, destacándose el trabajo de Rivière, quien aportó una definición y diferenciación dentro de las habilidades mentalistas. Por un lado, el carácter implícito como las expresiones faciales emocionales; y por otro, el carácter explícito como el lenguaje de estados mentales. Esas manifestaciones se harían evidentes en diferentes momentos evolutivos, se alterarían diferencialmente, implicarían niveles de acceso personal distintos y se relacionarían diferencialmente con otras competencias.

El poder comprender las expresiones emocionales es esencial para las interacciones sociales, lo que permite explicar y anticipar las acciones de los otros. A diferencia de otros procesos mentales, las emociones frecuentemente se hacen visibles a través de expresiones faciales. El darse cuenta de que las expresiones faciales tienden a revelar algo sobre el estado interno de las personas y atender a esas expresiones puede contribuir al desarrollo de habilidades mentalistas (Baron-Cohen et al., 1985).

Diferentes estudios con personas con autismo han reportado una atención reducida a las expresiones faciales de emociones, por ejemplo, en tareas donde tenían que clasificar o emparejar fotografías de emoción o no (Celani, Battachi, \& Arcidiacono, 1999) o formar pares de distintos modos de expresión emocional (Hobson, Houston \& Lee, 1988). Sin embargo, otros estudios no encontraron evidencia de una dificultad para atender a emociones en personas con autismo, particularmente cuando los participantes eran semejantes en cuanto a inteligencia verbal (Ozonoff, Pennington \& Rogers, 1990).

Begeer, Rieffe, Meerum Terwogt y Stockmann (2006) observaron que niños autistas de alto funcionamiento prestaban poca atención a las expresiones emocionales de otras personas en relación a un grupo control de niños con desarrollo normal en condiciones que los autores 
consideraban como neutrales, pero esa diferencia desaparecía si se requería realizar una decisión socialmente relevante. Ello sugiere que la atención a expresiones emocionales es influenciada por factores situacionales.

Otros investigadores se han centrado no en el reconocimiento de las emociones, sino en su predicción, para observar en qué medida las personas con autismo comprenden las causas de la emoción. En un estudio realizado por Baron-Cohen (1991) las personas con autismo eran capaces de reconocer la emoción de un personaje cuando era causada por la situación, y tenían un rendimiento semejante a un grupo de personas con retraso mental cuando debían predecir la emoción de un personaje conociendo su deseo. Sin embargo, mostraban un desempeño peor si lo que conocían era la creencia del personaje, tanto en relación a niños de 5 años con desarrollo normal como a personas con retraso mental. Además, las personas con autismo, también mostraron una dificultad en el reconocimiento de expresiones emocionales de sorpresa, en relación a las expresiones de felicidad o tristeza (Baron-Cohen, Spitz \& Cross, 1993). Ello parece indicar que las emociones simples se pueden comprender, pero no las emociones cognitivas o basadas en creencias.

Hadwin, Baron-Cohen, Howlin y Hill (2001) dieron a conocer un estudio en el que se propusieron evaluar si era posible enseñar a comprender estados mentales de emoción, creencia y ficción a personas con autismo. Los resultados mostraron que sí era posible enseñarles a pasar tareas que evalúan la comprensión de emociones y creencias. Sin embargo, encontraron que los efectos de la enseñanza no se generalizaban a otras tareas de dominios no enseñados específicamente. Los autores señalaron que quizás los niños pasaban las taras utilizando ciertas reglas en lugar de comprender auténticamente los conceptos implicados en las mismas.

Parece ser difícil dar instrucciones explícitas sobre la comprensión de expresiones emocionales en situaciones sociales que son ambiguas. Por lo que es un desafío poder facilitar experiencias sociales e interpersonales que hagan que se destaquen las expresiones emocionales, promoviendo su reconocimiento y comprensión.

\section{REFERENCIAS BIBLIOGRÁFICAS}

1. Abe, J. A., \& Izard, C. E. (1999). The developmental functions of emotions: An analysis in terms of Differential Emotions Theory. Cognition and Emotion, 13, 523 549.

2. American Psychological Association (APA) (2002). DSM-IV-TR. Manual diagnóstico y estadístico de los trastornos mentales. Texto revisado. Barcelona: Masson.

3. Baron-Cohen, S. (1991). Do people with autism understand what causes emotion? Child Development, 62, 385-395. 
4. Baron-Cohen, S., Leslie, A. M., \& Frith, U. (1985). Does the autistic child have a "theory of mind"? Cognition, 21, 37-46.

5. Baron-Cohen, S., Spitz, A., \& Cross, P. (1993). Can children with autism recognize surprise? Cognition and Emotion, 7, 507-516.

6. Begeer, S., Rieffe, C., Meerum Terwogt, M., \& Stockmann, L. (2006). Attention to facial emotional expressions in children with autism. Autism, 10, 37-51.

7. Cano, A. (1997). Modelos explicativos de la emoción. En E. G. Fernández-Abascal, Psicología General: Motivación y emoción (pp. 128-161). Madrid: Fundación Ramón Areces.

8. Celani, G., Battacchi, M. W., \& Arcidiacono, L. (1999). The understanding of the emotional meaning of facial expressions in people with autism. Journal of Autism and Developmental Disorders, 29, 57-66.

9. Fernández-Abascal, E. G. (2003). Procesamiento emocional. En E. G. FernándezAbascal, M. P. Jiménez Sánchez, y M. D. Martín Díaz, Emoción y Motivación, La adaptación humana (pp. 47-93). Madrid: Editorial Centro de Estudios Ramón Areces.

10. Fogel, A., Nwokah, E., Dedo, J. Y., Messinger, D., Dickson, K. L., Matusov, E., \& Holt, S. A. (1992). Social process theory of emotion: A dynamic systems approach. Social Development, 1(2), 122-142.

11. Hadwin, J., Baron-Cohen, S., Howlin, P., \& Hill, K. (2001). ¿Podemos enseñar a comprender emociones, creencias o ficciones a los niños autistas? En A. Rivière, y J. Martos (comps.). El tratamiento del autismo. Nuevas perspectivas (pp. 589-621). Madrid: IMSERSO.

12. Hobson, P. (1993). Understanding persons: the role of affect. En S. Baron-Cohen, H. Tager-Flusberg, \& D. J. Cohen (ed.), Understanding other minds: Perspectives from Autism (pp. 204-227). Oxford: Oxford University Press.

13. Hobson, P.; Houston, J., \& Lee, A. (1988). "What's in a face? The case of autism". British Journal of Psychology, 79, 441-453.

14. Izard, C. E. (1977). Human Emotions. New York: Plenum Press.

15. Kanner, 1. (1943). Autistic disturbances of affective contact. Nervous Child, 2, 217-250.

16. Keltner, D., \& Gross, J. J. (1999). Functional accounts of emotions. Cognition and Emotion, 13(5), 467-480.

17. Leslie, A. M. (2001). Theory of mind. En N. J. Smelser, \& P. B. Baltes (eds.), International Encyclopedia of the Social \& Behavioral Science (pp. 15652-15656). Oxford: Elsevier Science.

18. Ozonoff, S., Pennington, B., \& Rogers, S. J. (1990). Are there emotion perception deficits in young autistic children? Journal of Child Psychology and Psychiatry, 31, 343-363. 
19. Perner, J., \& Lang, B. (1999). Development of theory of mind and executive control. Trends in Cognitive Sciences, 3, 337-344.

20. Premack, D. G., \& Woodruff, G. (1978). Does the chimpanzee have a theory of mind? Behavioral and Brain Sciences, 1, 515-526.

21. Rivière, A. (2001). Tratamiento y definición del espectro autista I: Relaciones socials y comunicación. Tratamiento y definición del espectro autista II: Anticipación, flexibilidad y capacidades simbólicas. En A. Rivière, y J. Martos (comps.). El tratamiento del autismo. Nuevas perspectivas (pp. 61-160). Madrid: IMSERSO.

22. Rivière, A. (2004). El autismo y los trastornos generalizados del desarrollo. En A. Marchesi, C. Coll, y J. Palacios (comps.). Desarrollo psicológico y educación. 3. Trastornos del desarrollo y necesidades educativas especiales. Madrid: Alianza Editorial.

23. Rivière, A., \& Núñez, M. (2001). La mirada mental. 3. ${ }^{\text {a }}$ ed. Buenos Aires: Aique.

24. Saarni, C., Mumme, D. L., \& Campos, J. (1998). Emotional development: Action, communication, and understanding. En W. Damon (Series Ed.) \& N. Eisenberg (Vol. Ed.), Handbook of child psychology: Vol. 3. Social, emotional, and personality development (5th ed., pp. 237-309). New York: Wiley.

25. Sotillo, M. (2001). Teoría de la mente: Lugar de encuentro. En R. Rosas (comp.). La mente reconsiderada. En homenaje a Angel Rivière (pp. 121-139). Santiago: Psykhe.

26. Trevarthen, C. (1982). The primary motives for cooperative understanding. En G. Butterworth \& P. Light (eds.), Social cognition: Essays on the development of understanding (pp. 77-109). Brighton, Sussex: Harvester.

27. Valdez, D. (2001). Teoría de la mente y espectro autista. En D. Valdez (coord.), Autismo: enfoques actuales para padres y profesionales de la salud y la educación. Buenos Aires: Fundec.

28. Wimmer, H., \& Perner, J. (1983). Beliefs about beliefs: Representation and constraining function of wrong beliefs in young children's understanding of deception. Cognition, 13, 103-128. 